Louisiana State University

LSU Digital Commons

$1-1-2001$

\title{
The genetics of reproductive isolation and the potential for gene exchange between Drosophila Pseudoobscura and D. Persimilis via backcross hybrid males
}

\author{
Mohamed A.F. Noor \\ Louisiana State University \\ Katherine L. Grams \\ Louisiana State University \\ Lisa A. Bertucci \\ Louisiana State University \\ Yvette Almendarez \\ Louisiana State University \\ Jane Reiland \\ Louisiana State University
}

See next page for additional authors

Follow this and additional works at: https://digitalcommons.Isu.edu/biosci_pubs

\section{Recommended Citation}

Noor, M., Grams, K., Bertucci, L., Almendarez, Y., Reiland, J., \& Smith, K. (2001). The genetics of reproductive isolation and the potential for gene exchange between Drosophila Pseudoobscura and D. Persimilis via backcross hybrid males. Evolution, 55 (3), 512-521. https://doi.org/10.1111/ j.0014-3820.2001.tb00785.x

This Article is brought to you for free and open access by the Department of Biological Sciences at LSU Digital Commons. It has been accepted for inclusion in Faculty Publications by an authorized administrator of LSU Digital Commons. For more information, please contact ir@lsu.edu. 


\section{Authors}

Mohamed A.F. Noor, Katherine L. Grams, Lisa A. Bertucci, Yvette Almendarez, Jane Reiland, and Kelley R. Smith 


\title{
THE GENETICS OF REPRODUCTIVE ISOLATION AND THE POTENTIAL FOR GENE EXCHANGE BETWEEN DROSOPHILA PSEUDOOBSCURA AND D. PERSIMILIS VIA BACKCROSS HYBRID MALES
}

\author{
Mohamed A. F. Noor,${ }^{1}$ Katherine L. Grams, Lisa A. Bertucci, Yvette Almendarez, Jane Reiland, and \\ Kelley R. SMith \\ Department of Biological Sciences, Louisiana State University, Baton Rouge, Louisiana 70803 \\ ${ }^{1}$ E-mail:mnoor@lsu.edu
}

\begin{abstract}
Hybrid male sterility, hybrid inviability, sexual isolation, and a hybrid male courtship dysfunction reproductively isolate Drosophila pseudoobscura and D. persimilis. Previous studies of the genetic bases of these isolating mechanisms have yielded only limited information about how much and what areas of the genome are susceptible to interspecies introgression. We have examined the genetic basis of these barriers to gene exchange in several thousand backcross hybrid male progeny of these species using 14 codominant molecular genetic markers spanning the five chromosomes of these species, focusing particularly on the autosomes. Hybrid male sterility, hybrid inviability, and the hybrid male courtship dysfunction were all associated with $\mathrm{X}$-autosome interactions involving primarily the inverted regions on the left arm of the $\mathrm{X}$-chromosome and the center of the second chromosome. Sexual isolation from $D$. pseudoobscura females was primarily associated with the left arm of the X-chromosome, although both the right arm and the center of the second chromosome also contributed to it. Sexual isolation from D. persimilis females was primarily associated with the second chromosome. The absence of isolating mechanisms being associated with many autosomal regions, including some large inverted regions that separate the strains, suggests that these phenotypes may not be caused by genes spread throughout the genome. We suggest that gene flow between these species via hybrid males may be possible at loci spread across much of the autosomes.
\end{abstract}

Key words._Drosophila, hybrid sterility, introgression, reproductive isolation, speciation.

Received July 6, 2000. Accepted October 8, 2000.

Speciation has generally been defined as the evolution of barriers to gene exchange (isolating mechanisms) between taxa. Thus, over the past half-century, many studies of speciation genetics have focused on determining the genetic basis of these barriers to gene flow. Several general observations have emerged, particularly with regard to mating discrimination and hybrid sterility in Drosophila species. The Xchromosome has been suggested to have a disproportionate role in hybrid male sterility (Charlesworth et al. 1987; Coyne and Orr 1989), although more recent studies have questioned either the existence or the extent of this disproportionate role (Hollocher and Wu 1996; True et al. 1996). Both barriers generally appear to involve multiple genes, and few major gene effects have been identified (Wu and Palopoli 1994; Maside and Naveira 1996; Coyne and Orr 1998; Hollocher 1998; Ritchie and Phillips 1998; Ting et al. 1998). Often, almost every genetic marker used in a mapping study is significantly associated with the particular barrier being examined (e.g., Orr 1987; Coyne 1989). However, these studies and particularly those of mating discrimination have been limited in their scope because only a handful of genetic markers were used (see Coyne and Orr 1998). Thus, it has not been possible to determine whether these barriers are associated with genes spread across the genome or with localized regions of the genome.

An exciting area in the study of speciation is how, when reproductive isolation is not complete, certain parts of the genome can readily cross species boundaries while others cannot (e.g., see Wang et al. 1997; Butlin 1998; Rieseberg et al. 1999; Jiang et al. 2000). In hybridizing species, regions of the genome linked with barriers to gene exchange such as hybrid sterility or sexual isolation should penetrate poorly, whereas unlinked or loosely linked regions of the genome should penetrate more easily. If much of the genome is strongly associated with such barriers to gene exchange, then little interspecies introgression could ever occur. The low marker density of many previous studies makes it difficult to determine whether much or little of genomes are associated with such barriers and, therefore, the potential for interspecies introgression.

The genetic bases of various barriers to gene exchange have been investigated in Drosophila pseudoobscura and $D$. persimilis (Dobzhansky 1936; Tan 1946; Weisbrot 1963; Wu and Beckenbach 1983; Orr 1987, 1989; Noor 1997b). These species are isolated by $F_{1}$ hybrid male sterility, backcross hybrid inviability, strong species discrimination exercised by females, and an $F_{1}$ hybrid male courtship dysfunction. Nonetheless, they do hybridize at very low frequencies in nature because $F_{1}$ hybrids have been captured in the field (Dobzhansky 1973; Powell 1983), and introgression has been detected in one region of the fourth chromosome (Wang et al. 1997). These species have had a central role in studies of speciation, and the question of whether or how much gene exchange between them is possible has been debated (Dobzhansky 1973; Powell 1983, 1991; Kulathinal and Singh 2000; Noor et al. 2000a).

Previous genetic studies of the barriers to gene exchange that separate these species via males have focused primarily or exclusively on regions of the X-chromosome (see Table 1). Orr's (1987) study of hybrid sterility examined one marker per major autosome, and Noor's (1997b) studies of sexual isolation and hybrid male courtship dysfunction only examined one region of the third chromosome. Thus, much of the genome of these species has never been investigated with 
TABle 1. Summary of studies of reproductive isolation in hybrid males of Drosophila pseudoobscura and D. persimilis.

\begin{tabular}{|c|c|c|c|c|}
\hline Study & Phenotype & $\begin{array}{l}\text { No. X- } \\
\text { chromosome } \\
\text { regions } \\
\text { tested }\end{array}$ & $\begin{array}{l}\text { No. } \\
\text { autosome } \\
\text { regions } \\
\text { tested }\end{array}$ & Effect detected \\
\hline Weisbrot (1963) & inviability & 1 & 3 & significant effect of chromosome 3 \\
\hline Noor (1997b) & $\begin{array}{l}\text { sexual isolation } \\
\text { courtship dysfunction }\end{array}$ & 2 & 1 & $\begin{array}{l}\text { multiple X-linked factors suggested } \\
\text { no autosomal effect detected }\end{array}$ \\
\hline
\end{tabular}

regard to associations with barriers to gene exchange. Further, a recent power analysis of previous studies of sexual isolation suggested that Noor's (1997b) study (and many others) lacked sufficient power to detect potentially large effects of linked genomic regions due to the small sample size used ( $n=100$; Noor and Smith 2000).

Here, we expand on these previous studies to genetically map the four known barriers to gene exchange in backcross hybrid males of $D$. pseudoobscura and $D$. persimilis: hybrid male sterility, hybrid inviability, the hybrid male courtship dysfunction, and sexual isolation. Noor et al. (2000b) recently isolated numerous molecular markers (microsatellites and restriction fragment length polymorphisms [RFLPs]) from $D$. pseudoobscura. We evaluate the association between 14 molecular markers (12 on the autosomes: an increase of four to 12 times over recent studies) and the four barriers to gene exchange. Specifically, we seek to determine how much and what regions of the genome have the potential to introgress between these species via backcross hybrid males. We are also interested in the genetic basis of these barriers to gene exchange. To address these questions, we have concentrated on producing very large sample sizes rather than assaying many more genetic markers because we are interested in potentially weak effects associated with these genomic regions.

\section{Materials And Methods}

\section{Background, Crosses, and Handling of Flies}

Drosophila pseudoobscura and D. persimilis have a metacentric X-chromosome and four telocentric autosomes. These species are separated by paracentric inversions on parts of four of their six chromosome arms spanning various lengths: six cytological bands on the left arm of the X-chromosome (XL), 11 cytological bands on the right arm of the X-chromosome (XR), five cytological bands in the center of the second chromosome, and various arrangements on the third chromosome (Tan 1935). However, recombination still occurs across the long, uninverted regions of these chromosomes (Sturtevant and Dobzhansky 1936; Dobzhansky and Sturtevant 1938; Noor and Smith 2000). Thus, genes affecting barriers to gene exchange can be identified across uninverted regions, but cannot be localized to particular locations within the inversions that separate these species.
Two strains were used in the backcross mapping experiment, D. pseudoobscura Flagstaff 1993 (third chromosome arrangement " Arrowhead") and D. persimilis Mount St. Helena 1993 (third chromosome arrangement "Standard"'). These chromosome arrangements differ by a single inversion along the middle of this chromosome spanning seven cytological bands, and they appear to be the most common within these species. Both strains have been maintained in the laboratory for several years and used previously in various other studies of mating discrimination (e.g., Noor 1995, 1997b). To identify strain-specific markers, we genotyped several individuals from numerous culture bottles at all surveyed marker loci.

Females from the D. pseudoobscura strain were crossed to males of the $D$. persimilis strain, and the resultant fertile $\mathrm{F}_{1}$ females were backcrossed to males of each parental line. All crosses were carried out at $20 \pm 1{ }^{\circ} \mathrm{C}, 85 \%$ relative humidity, on standard sugar/yeast/agar medium. Backcross hybrid males were designated as BCps if they were offspring of $D$. pseudoobscura fathers or BCper if they were offspring of $D$. persimilis fathers. Bottles were cleared, and virgin backcross hybrid males and virgin pure species females were harvested $7 \mathrm{~h}$ later. The flies were then aged for 7 days in groups of five to 20 individuals. On day 7 after eclosion, males were separated into individual vials for 1 day to reduce crowdingmediated courtship inhibition (Noor 1997a).

On day 8 , single females were aspirated into each vial containing a backcross hybrid male. Fly pairs were observed for $5 \mathrm{~min}$ after the onset of courtship or $5 \mathrm{~min}$ in total if no courtship occurred. Courtship was defined as wing vibration or attempted copulation by the male (Noor 1997b). Male courtship intensity and probability do not depend on the species of the female they are presented in these taxa (Noor 1996), so we have not separated the courtship data with regard to female species. If no courtship occurred, the fly was designated as a noncourter and removed. If courtship did occur, we recorded whether the fly was successful at copulating with the female for at least $30 \mathrm{sec}$ during the observation period. Males that failed to achieve a 30 -sec copulation were considered to be discriminated against by the female and were scored with a 0 . Males that successfully copulated within their first two attempts were scored as 2. Other males that copulated within 5 min of courtship initiation but after two 

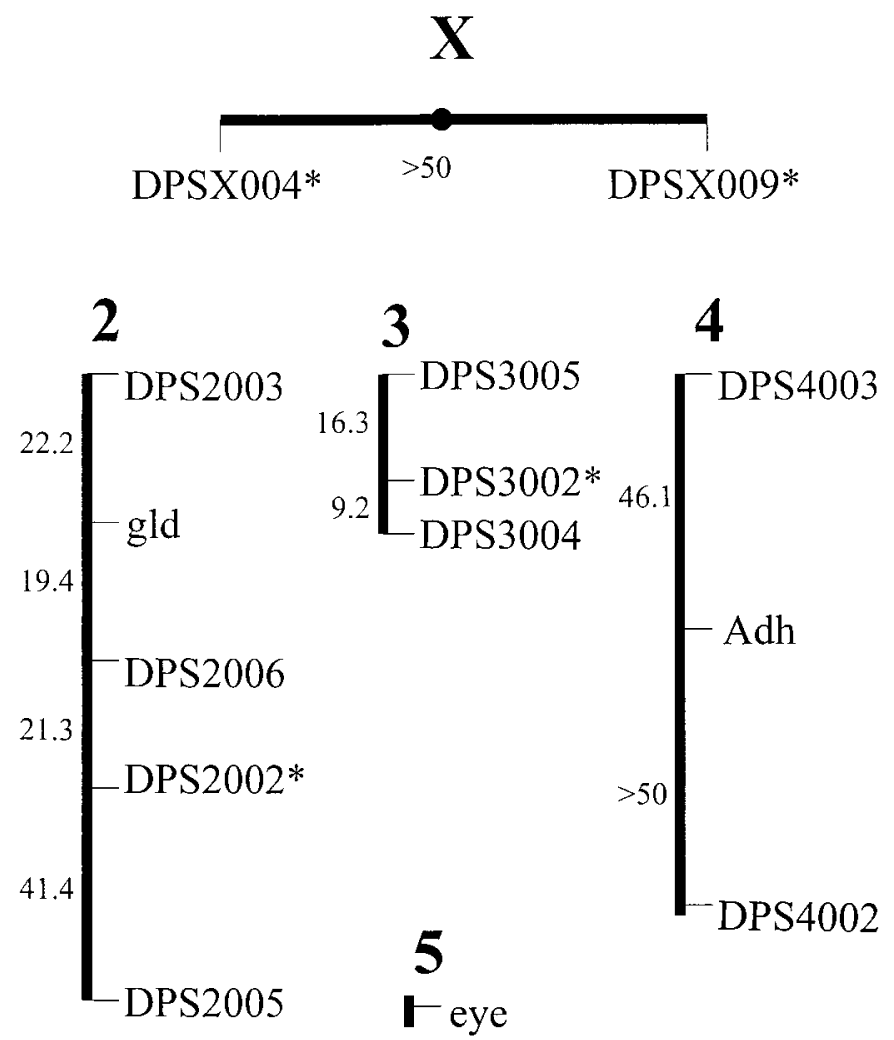

FIG. 1. Recombinational map (in Kosambi centimorgans) of molecular markers on the five chromosomes in backcross hybrids of $D$. pseudoobscura and D. persimilis. The centromeres of the autosomes are hypothesized to be at the ends closest to the top of the figure. DPS4002 is unlinked to the other two fourth chromosome markers, and DPSX004 and DPSX009 are unlinked to each other. Markers indicated with an asterisk are linked to strain-specific inversions.

attempts were scored as 1 . All observations were performed between 0700 and $1100 \mathrm{~h}$, and each male and female were only used once.

Following courtship assays, male fertility was assayed by dissection of testes in insect Ringer's solution using the method of Coyne (1984). A male was scored as fertile (1) if he had any motile sperm and sterile (0) if no motile sperm were observed. Males were then frozen in labeled 0.6-ml microcentrifuge tubes. All methods were identical for assays of pure species flies except that no backcross was performed.

\section{Molecular Markers}

Most of the microsatellites and RFLPs used in this study have been described in detail elsewhere (Noor and Smith 2000; Noor et al. 2000b), including both their variability in natural populations and recombinational distances between markers in species hybrids (see also Fig. 1). In short, two markers were used on the X-chromosome, one on each chromosome arm and linked to the species-specific inversions: DPSX004 and DPSX009. A previous study suggested that little recombination occurs along these arms outside of the inversions, although the chromosome arms recombine from one another freely (Noor and Smith 2000). Five markers were used along the second chromosome: DPS2005, DPS2002 (linked to the inversion), DPS2006, gld, and DPS2003. The average spacing of these markers was approximately $25 \mathrm{cM}$. Three markers were used along the third chromosome: DPS3005, DPS3002 (linked to the inversion), and DPS3004, which are spaced an average of $13 \mathrm{cM}$ apart. Three markers were used along the fourth chromosome: DPS4003 and Adh, which are approximately $46 \mathrm{cM}$ apart, and DPS4002, which recombines freely from the other two markers. Finally, we developed a new RFLP marker in the eyeless gene on the nonrecombining fifth (dot) chromosome of this species. A 152-bp segment was amplified using primers 5'-ACTTCACAGGTTGTACAGTAATGTGTACC - 3' and 5'-GTAGGTCGAGGCTATGAGGTCG-3'. This product was then cut using HinfI, which produced a 100-bp fragment for the $D$. pseudoobscura allele and a 97-bp fragment for the $D$. persimilis allele.

The behavioral traits we are examining may have a low heritability (e.g., Williams et al. 2001), making an individual's phenotype a poor indicator of his genotype and necessitating our use of very large sample sizes. We also needed large sample sizes because the characters are often binary (mated vs. did not mate; Noor and Smith 2000) and because weak effects of particular genomic regions may also prevent gene exchange. Darvasi and Soller (1994) suggested that markers spaced every 20-30 cM give a high probability of quantitative trait locus (QTL) detection, although not necessarily precise position or effect. Thus, our coverage of the second, third, and fifth chromosomes is satisfactory for detection of associations with the barriers to gene exchange. The X-chromosome has already been studied extensively ( $\mathrm{Wu}$ and Beckenbach 1983), so we have not focused on it here.

Microsatellites were assayed on agarose gels if the difference in size between the D. pseudoobscura and D. persimilis line alleles exceeded $10 \mathrm{bp}$ or on acrylamide gels for smaller size differences. For microsatellite assays on acrylamide gels, one primer was ordered with an M13 tail at the $5^{\prime}$ end, and polymerase chain reaction (PCR) was performed in a $10-\mu 1$ reaction volume with 0.5 picomoles of each primer, 0.4 picomoles fluorescent dye labeled M13, $200 \mu \mathrm{M}$ dNTPs, $1 \mu \mathrm{l}$ $10 \times$ buffer $(100 \mathrm{mM}$ Tris $\mathrm{pH} 8.3,500 \mathrm{mM} \mathrm{KCl}, 15 \mathrm{mM}$ $\mathrm{MgCl}_{2}$ ), $1 \mathrm{U}$ Taq polymerase, and $1 \mu \mathrm{l}$ from a 50 - $\mu$ l single fly squish prep (Gloor and Engels 1992). PCR was executed using a touchdown cycle (Palumbi 1996). Following PCR, 3 $\mu l$ of LiCor (Lincoln, NE) stopping buffer was added to the reactions, and $1 \mu \mathrm{l}$ of the PCR reaction was loaded onto an acrylamide gel (National Diagnostics Sequagel, Atlanta, GA) on a LiCor 4200 DNA sequencer for visualization. Individual males were scored as homozygous for a strain-specific allele or heterozygous for the alleles of the two strains. The eyeless RFLP was also assayed on acrylamide gels.

Microsatellite assays on agarose gels followed a similar PCR protocol except that primers were not labeled and 5 picomoles of each primer were used per reaction. Following PCR, $3 \mu \mathrm{l}$ of Ficoll (Sigma Chemical Co., St. Louis, MO) loading dye was added to the reactions, and all $13 \mu$ l were loaded into a $2 \%$ agarose gel. The $A d h$ RFLP was also assayed on agarose gels.

\section{Allele Segregation Ratios}

We evaluated the allelic segregation ratios at each of the markers used in this study. Alleles present the $F_{1}$ hybrid 
TABle 2. Fertility, courtship, and mating success of Drosophila pseudoobscura, D. persimilis, and their backcross hybrids. Numbers in parentheses indicate the sample size used for deriving the particular figure. See Methods for scoring details.

\begin{tabular}{|c|c|c|c|c|c|c|c|c|}
\hline \multirow[b]{2}{*}{ Males } & \multirow[b]{2}{*}{ Fertile } & \multirow[b]{2}{*}{ Courted } & \multicolumn{3}{|c|}{ Mated D. pseudoobscura females } & \multicolumn{3}{|c|}{ Mated $D$. persimilis females } \\
\hline & & & Overall & $\begin{array}{c}\text { Two } \\
\text { attempts }\end{array}$ & Score & Overall & $\begin{array}{c}\text { Two } \\
\text { attempts }\end{array}$ & Score \\
\hline D. persimilis & $100 \%(137)$ & $77 \%(298)$ & $45 \%(110)$ & $30 \%$ & 0.755 & $84 \%(120)$ & $78 \%$ & 1.617 \\
\hline D. pseudoobscura & $98 \%(164)$ & $89 \%(245)$ & $95 \%(112)$ & $88 \%$ & 1.830 & $54 \%(106)$ & $30 \%$ & 0.840 \\
\hline BCper & $49 \%(1047)$ & $83 \%(1282)$ & $68 \%(512)$ & $63 \%$ & 1.307 & $68 \%(549)$ & $59 \%$ & 1.273 \\
\hline $\mathrm{BCps}$ & $39 \%(1147)$ & $70 \%(1482)$ & $89 \%(503)$ & $84 \%$ & 1.730 & $55 \%(503)$ & $41 \%$ & 0.962 \\
\hline
\end{tabular}

female should be found in the backcross male offspring at a frequency of $50 \%$. Significant deviations from this expectation would suggest either hybrid inviability associated with a particular allele or meiotic drive altering the segregation ratio among the eggs that form the backcross hybrid males. As hybrid male inviability has been reported in these species (Weisbrot 1963), we presume this to be a more likely cause of such deviations from expectation (see Discussion for further support).

For each marker, we evaluated whether there was significant under- or overrepresentation of alleles at particular loci across backcross hybrid males using a chi-square test against an expectation of $50 \%$. The two backcrosses were analyzed independently. Deviations reported as statistically significant had $\alpha<0.05$ after a Bonferroni correction for 14 markers (Rice 1989), resulting in $\alpha<0.003$ in practice.

\section{Genetic Mapping}

More than 2500 backcross hybrid males were genotyped for each marker: 1282 BCps males and 1482 BCper males (Table 2). For each trait, the two backcross directions were analyzed separately. Given that our data are binary rather than continuous and because of the spacing of our markers, standard programs for interval mapping or composite interval mapping could not localize putative QTLs within the intervals between our markers. We present our data in two ways. First, we present the raw effects of alternate alleles at each locus on the phenotypes being examined. For example, if $67 \%$ of males bearing one allele at a locus were fertile and only $2 \%$ of males bearing the alternate allele were fertile, we present these two numbers. This clearly illustrates the effects associated with each locus. Second, single marker regression analyses were performed using the QTL Cartographer (Basten et al. 1999) suite of programs (LRMapQTL, in particular). Using results from these analyses, we present both the proportion of the observed variation attributable to the marker $\left(r^{2}\right)$ and its statistical significance following 1000 permutations. We acknowledge that estimates of effect are likely to be biased downward from single marker analyses due to recombination, but we present $r^{2}$ to illustrate approximate minimum magnitudes of effect. Associations reported as statistically significant had $\alpha<0.01$. Only statistically significant associations are reported in the tables.

Although we have performed backcrosses in both directions, we cannot estimate additive and dominance effects associated with the markers we studied, as might have been possible with an $\mathrm{F}_{2}$ cross. In the two backcrosses, no offspring were formed that are homozygous for D. pseudoobscura al- leles at one locus but homozygous for $D$. persimilis alleles at another, which may have been possible if an $\mathrm{F}_{2}$ cross could have been performed. If we were to combine the data from the two backcrosses and analyze them as an $\mathrm{F}_{2}$ cross, we would overestimate effects associated with particular regions if any unlinked or loosely linked loci contributed to the phenotypes being studied. Thus, the two backcrosses must be analyzed separately.

For the mapping of the hybrid courtship dysfunction and hybrid male sterility, we also tested for weak QTLs and for interactions between $\mathrm{X}$-chromosomal and autosomal regions using three methods. First, we used the CET protocol of Doerge and Churchill (1996) to identify potentially weak quantitative trait loci. When a strong effect was associated with a particular marker, the backcrosses were stratified into the separate classes for that marker and a reanalysis was performed with 1000 permutations. This procedure was repeated until no additional significant marker associations were detected. Second, we limited the dataset to only those backcross males bearing both X-chromosomal markers from the species that corresponds with the majority of the autosomal genotype (e.g., D. persimilis X-chromosome markers for BCper males). This stratification should make epistatic effects between X-chromosomal factors and heterozygous autosomal factors easier to detect. Finally, we performed multiple regressions of the phenotypes on X-chromosomal markers, autosomal markers, and their interactions (see Gurganus et al. 1999) to identify particular interacting regions. Because all of these procedures yielded essentially the same results, we report only the latter two.

No effects were detected on the fourth or fifth chromosomes for any phenotype, so these chromosomes are not presented in the tables or figures.

\section{RESUlTS \\ Pure Species Pairings}

Drosophila pseudoobscura and D. persimilis males were nearly always fertile (Table 2). In addition, a large fraction of them generally courted any females they were presented, although the $D$. persimilis males $(77 \%)$ were poorer than $D$. pseudoobscura males (89\%) in that regard. Mating success was typically very high within species (84-95\%) and substantially lower in interspecies pairings (45-54\%).

\section{Genetics of Courtship Dysfunction}

As previously documented (Noor 1997b), many backcross males failed to court the female they were presented (Table 
TABLE 3. Coefficients of determination $\left(r^{2}\right)$ of associations between marker loci and phenotypes in backcross hybrid males of Drosophila pseudoobscura and $D$. persimilis. Only values statistically significant following Bonferroni correction are presented.

\begin{tabular}{|c|c|c|c|c|c|c|c|c|c|c|}
\hline & DPSX004 & DPSX009 & DPS2005 & DPS2002 & DPS2006 & gld & DPS2003 & DPS3004 & DPS3002 & DPS300 \\
\hline \multicolumn{11}{|l|}{$\overline{\mathrm{BCps}}$} \\
\hline Courtship & 0.207 & 0.060 & ns & 0.006 & ns & 0.008 & $\mathrm{~ns}$ & ns & ns & ns \\
\hline Sterility & 0.430 & 0.014 & 0.007 & 0.050 & 0.032 & $\mathrm{~ns}$ & $\mathrm{~ns}$ & ns & ns & ns \\
\hline Mate $D . p e r$ & 0.019 & $\mathrm{~ns}$ & ns & 0.026 & ns & ns & $\mathrm{ns}$ & ns & ns & ns \\
\hline \multicolumn{11}{|l|}{ BCps limited } \\
\hline Sterility & - & - & 0.028 & 0.363 & 0.141 & 0.019 & ns & ns & ns & ns \\
\hline Courtship & 0.026 & $\mathrm{~ns}$ & ns & ns & ns & ns & $\mathrm{ns}$ & ns & ns & ns \\
\hline Sterility & 0.274 & 0.015 & ns & 0.019 & ns & ns & $\mathrm{ns}$ & ns & ns & ns \\
\hline Mate D. ps & 0.137 & 0.017 & ns & 0.036 & ns & ns & $\mathrm{ns}$ & ns & ns & ns \\
\hline Mate $D . p e r$ & ns & $\mathrm{ns}$ & ns & 0.047 & ns & ns & ns & ns & ns & ns \\
\hline \multicolumn{11}{|l|}{ BCper limited } \\
\hline Courtship & - & - & ns & 0.054 & 0.043 & ns & $\mathrm{ns}$ & ns & ns & ns \\
\hline
\end{tabular}

2). The fraction of BCps males that failed to court was significantly larger than the fractions of $D$. persimilis and $D$. pseudoobscura males that failed to court (Fisher's exact tests, $P=0.0015$ and $P<0.0001$, respectively). Noor (1997b) suggested that this phenotype may be a behavioral manifestation of Haldane's rule, as the heterogametic sex experiences a dysfunction only in hybrids. Because this dysfunction was manifested in $\mathrm{F}_{1}$ hybrids bearing $D$. persimilis mothers, we predict that $\mathrm{X}$-chromosomal loci from $D$. persimilis are interacting with either heterozygous $D$. pseudoobscura autosomal loci or the D. pseudoobscura Y-chromosome. If the $\mathrm{X}$-chromosome interacts with the autosomes to produce this dysfunction, X-chromosomal effects should be observed in both backcrosses but should be most pronounced in BCps males because they are never homozygous for $D$. persimilis autosomal regions. In addition, autosomal effects should be detected in backcross males, and particularly in BCper males.
In contrast, if $\mathrm{X}-\mathrm{Y}$ chromosome interactions cause this dysfunction in hybrids, X-chromosomal effects should be detected only in BCps males and no autosomal effects should be noted.

We observed a strong effect associated with the X-chromosomal markers on courtship in males of both backcrosses, and particularly in BCps males (Tables 3,4). This finding is consistent with the $\mathrm{X}$-autosome interaction hypothesis for this courtship dysfunction. The marker DPSX004 was associated with over $20 \%$ of the variance in this phenotype in BCps males, and the marker DPSX009 was associated with an additional $6 \%(P<0.001$ in both cases $)$. In both cases, bearing the $D$. persimilis allele reduced the probability of courtship in BCps males. Weak, although detectable, effects of autosomal loci were found in BCps males both DPS2002 and $\operatorname{gld}(P=0.001$ and $P<0.001$, respectively), each accounting for under $1 \%$ of the variance in the character. Bear-

TABLE 4. Effects of alternate alleles on backcross hybrid males of Drosophila pseudoobscura and D. persimilis. The first number in each entry is the value for males bearing only the homospecific allele (e.g., the D. pseudoobscura allele in BCps males) and the second is for males bearing one heterospecific allele. Mating percentages compare proportions that copulated within the first two attempts versus those that did not copulate within the first two attempts.

\begin{tabular}{|c|c|c|c|c|c|c|c|c|c|c|}
\hline & DPSX004 & DPSX009 & DPS2005 & DPS2002 & DPS2006 & gld & $\begin{array}{l}D P S- \\
2003\end{array}$ & DPS3004 & DPS3002 & DPS3005 \\
\hline \multicolumn{11}{|l|}{ BCps } \\
\hline$\%$ Courting & $85.4 / 42.2$ & $78.9 / 56.0$ & ns & $64.3 / 71.3$ & ns & $63.7 / 72.1$ & ns & ns & ns & ns \\
\hline$\%$ Mate $D . p s$ & $89.2 / 69.8$ & $88.7 / 77.2$ & ns & ns & $\mathrm{ns}$ & ns & $\mathrm{ns}$ & $\mathrm{ns}$ & $\mathrm{ns}$ & ns \\
\hline$\%$ Mate $D$. per & $35.9 / 57.1$ & $\mathrm{~ns}$ & ns & $33.0 / 46.0$ & $\mathrm{~ns}$ & ns & $\mathrm{ns}$ & ns & $\mathrm{ns}$ & ns \\
\hline \multicolumn{11}{|l|}{ BCps limited } \\
\hline$\%$ Courting & $78.0 / 90.2$ & $\mathrm{~ns}$ & ns & ns & $\mathrm{ns}$ & ns & $\mathrm{ns}$ & ns & $\mathrm{ns}$ & ns \\
\hline$\%$ Fertile & $69.7 / 16.3$ & $54.7 / 42.5$ & ns & $56.9 / 43.1$ & $\mathrm{~ns}$ & ns & $\mathrm{ns}$ & ns & $\mathrm{ns}$ & $\mathrm{ns}$ \\
\hline$\%$ Mate $D . p s$ & $45.5 / 79.8$ & $57.5 / 68.2$ & ns & $52.4 / 70.4$ & $\mathrm{~ns}$ & ns & $\mathrm{ns}$ & ns & $\mathrm{ns}$ & $\mathrm{ns}$ \\
\hline$\%$ Mate D. per & ns & $\mathrm{ns}$ & ns & $71.2 / 49.3$ & $\mathrm{~ns}$ & ns & $\mathrm{ns}$ & ns & ns & ns \\
\hline \multicolumn{11}{|l|}{ BCper limited } \\
\hline
\end{tabular}


ing the $D$. persimilis allele at these loci slightly increased the probability of courtship in BCps males. We did not initially detect any autosomal QTLs affecting courtship in BCper males.

If autosomal loci interact with the $D$. persimilis X-chromosome to produce the courtship dysfunction, these autosomal factors should be most detectable in BCper males when the $\mathrm{X}$-chromosome is derived from $D$. persimilis. Thus, we limited our dataset to those individuals bearing both $\mathrm{X}$-chromosomal markers (and therefore both inverted regions) from D. persimilis and surveyed the autosomes for QTLs affecting the courtship dysfunction in BCper males again. By doing this, we were able to detect a highly significant $(P<0.001)$ association on the second chromosome near DPS2002 and DPS2006, which explained over $5 \%$ of the observed variation (Tables 3, 4).

To more precisely document that this courtship dysfunction results from an interaction between $\mathrm{X}$-chromosomal loci and the region of DPS2002, we performed a multiple regression of courtship on DPSX004, DPS2002, and the interaction of these two regions (see Gurganus et al. 1999). We were able to identify both a significant main effect of the DPSX004 region and a significant interaction between the two regions (DPSX004, $P<0.0001 ;$ DPS2002, $P=0.43$; DPSX004 $\times$ DPS2002, $P=0.0003$ ) on whether BCper males courted.

\section{Genetics of Hybrid Sterility}

More than half of the backcross males surveyed did not possess any motile sperm (Table 2), a strong contrast to the high fertility of pure species males. As in previous studies (Dobzhansky 1936; Orr 1987), we found a very strong and highly significant effect of the left arm of the X-chromosome on this hybrid sterility, explaining $43.0 \%$ and $27.3 \%$ of the variation in hybrid male sterility in BCps and BCper males, respectively (Tables 3,4 ). The right arm of the X-chromosome also contributed statistically significant but much weaker effects, explaining $1.4 \%$ and $1.5 \%$ of the variation in BCps and BCper males $(P<0.001$ for all analyses $)$. Counterintuitively, the D. persimilis allele at DPSX009 enhanced fertility slightly in BCps males. This effect was still apparent when both DPSX004 and DPSX009 genotypes were considered together - the BCps males most likely to be fertile bore the D. pseudoobscura allele at DPSX004 and the D. persimilis allele at DPSX009.

Again, an autosomal effect was detected in the vicinity of DPS2002 in both backcrosses, accounting for $1-5 \%$ of the variation in each case $(P<0.001)$. No other autosomal regions appeared to independently contribute to hybrid sterility in our first analysis.

As above, we limited our analyses to just those individuals in the two backcrosses that bore homospecific X-chromosome arms (i.e., the X-chromosome arms borne by the father of the backcross males). This was done to reduce the variation resulting from the very large X-chromosomal effects observed in the two backcrosses. We did not survey males bearing the heterospecific X-chromosome arms because these males are almost invariably sterile. When we limited our dataset to males bearing both homospecific X-chromosome arms, the QTL(s) near DPS2002 were more apparent, asso- ciated with $19.8 \%$ and $36.3 \%$ of the observed variation in BCper and BCps males, respectively (Tables 3, 4). The magnitude of the association in BCps males was especially strong: the difference in fertility between those bearing a $D$. pseudoobscura allele versus a $D$. persimilis allele at this locus was nearly $60 \%$. A second QTL was also detected on the third chromosome near DPS3004, accounting for approximately $4 \%$ of this autosomal variation.

To more precisely identify epistatic interactions, we performed a multiple regression of fertility on DPSX004, DPS2002, DPS3004, and the interaction of X-chromosomal and autosomal regions (see Gurganus et al. 1999). By doing this, we were able to identify significant interactions between $\mathrm{X}$-chromosomal and both autosomal regions on backcross male fertility (BCper: DPSX004, $P<0.0001$; DPS2002, $P$ $=0.15 ;$ DPS3004, $P=0.14 ;$ DPSX004 $\times$ DPS2002, $P<$ $0.0001 ;$ DPSX004 $\times$ DPS3004, $P=0.0041$; BCps: DPSX004, $P<0.0001$; DPS2002, $P<0.0001 ; D P S 3004, P=0.91$; DPSX004 $\times$ DPS2002, $P<0.0001 ; D P S X 004 \times$ DPS3004, $P=0.51)$.

\section{Demographics of Hybrid Sterility}

A recent study suggested that some hybrid males may develop motile sperm more slowly than pure species males (Maside et al. 1998), such that males scored as sterile at one age may be scored at fertile if they were assayed at a later time. Given that we have studied the genetics of sterility in hybrids of $D$. pseudoobscura and $D$. persimilis, we need to ensure that males that are scored as sterile at 8 days would not have been later scored as fertile. To this end, we surveyed the proportions of BCper males that possessed motile sperm at 11 days and 15 days after eclosion and compared these with the proportions that we surveyed at 8 days after eclosion. All surveyed males eclosed from the same media bottles.

We found no significant difference in sperm motility between males 8 days versus 11 days after eclosion ( 8 days, $n$ $=257,55.6 \%$ fertile; 11 days, $n=229,52.0 \%$ fertile; $\chi^{2}=$ $0.70, P=0.4)$. We also found no significant difference in sperm motility between males 8 days versus 15 days after eclosion ( 8 days, $n=284,48.4 \%$ fertile; 15 days, $n=260$, $52.9 \%$ fertile; $\left.\chi^{2}=0.99, P=0.3\right)$. Paired comparisons using males that hatched on a particular day produced identical results. Fertility does not increase with increasing age in backcross hybrids of $D$. pseudoobscura and $D$. persimilis beyond 8 days after eclosion.

\section{Genetics of Hybrid Inviability}

Among BCps males, only two markers had segregation ratios significantly different from 50\%: DPSX004 (\% D. persimilis allele: $40.8, n=1442, P<0.001)$ and DPS2002 $(\%$ $D$. persimilis allele: $54.1, n=1439, P=0.002)$. Among BCper males, three markers deviated from expected segregation ratios significantly: DPSX004 (\% D. persimilis allele: 58.9, $n=1221, P<0.001)$, DPSX009 (\% D. persimilis allele: 56.6, $n=1209, P<0.001$ ), and DPS2002 (\% D. persimilis allele: $43.4, n=1175, P<0.001)$.

Given that the segregation patterns of the X-chromosomal and autosomal loci are distorted in opposite directions, we also tested for interactions between these genomic regions 
contributing to hybrid inviability. We used a chi-square test to compare the segregation ratio at the DPS2002 locus when both DPSX004 and DPSX009 bore a D. pseudoobscura allele versus when the two X-chromosomal loci bore a $D$. persimilis allele. A significant chi-square value would suggest that loci in the region of DPS2002 interact with loci in one or both of the X-chromosomal regions to cause the hybrid inviability found in the backcross males. Among BCps males bearing both X-chromosomal alleles from D. pseudoobscura, 232 bore the $D$. persimilis allele at DPS2002 and 216 bore the $D$. pseudoobscura allele. Among BCps males bearing both Xchromosomal alleles from $D$. persimilis, 172 bore the $D$. persimilis allele at DPS2002 and 128 bore the D. pseudoobscura allele. This difference was not statistically significant $(P=$ 0.15). Among BCper males bearing both X-chromosomal alleles from $D$. pseudoobscura, 74 bore the $D$. persimilis allele at DPS2002 and 132 bore the D. pseudoobscura allele. Among BCper males bearing both $\mathrm{X}$-chromosomal alleles from $D$. persimilis, 190 bore the D. persimilis allele at DPS2002 and 195 bore the $D$. pseudoobscura allele. This difference was statistically significant $(P=0.0018)$. The BCper genotype that was underrepresented bore a homozygous $D$. persimilis DPS2002 genotype and a hemizygous D. pseudoobscura Xchromosome.

\section{Genetics of Sexual Isolation and Mating Success}

\section{Drosophila pseudoobscura females}

Both arms of the X-chromosome contributed to backcross male sexual isolation from D. pseudoobscura females (BCper: DPSX004, $P<0.001$; DPSX009, $P=0.003$; BCps: DPSX004, $P<0.001$; DPSX009, $P<0.001$; Tables 3, 4). Unlike the previous genetic study (Noor 1997b), we found a larger effect of the left arm of the X-chromosome than the right arm, particularly among BCper males. We detected a significant association of the region of DPS2002 with mating success to D. pseudoobscura females in BCper males $(P<0.001)$, but not in BCps males $(P=0.45)$. Thus, the $D$. pseudoobscura autosomal factor(s) contributing to hybrid male mating success to females of that species appear to be dominant.

\section{Drosophila persimilis females}

The left arm of the X-chromosome was significantly associated with $\mathrm{BCps}$ male mating success to $D$. persimilis females $(P=0.006)$, but this region was not significantly associated with mating success in BCper males. The region of $D P S 2002$ had a significant effect on sexual isolation from $D$. persimilis females in both backcrosses (BCps, $P=0.001$; Bcper, $P<0.001$ ), each accounting for less than $5 \%$ of the observed variation.

\section{Discussion}

Using 14 microsatellite markers, we have genetically dissected four barriers to gene exchange in more than 2500 backcross hybrid males of $D$. pseudoobscura and D. persimilis: hybrid sterility, hybrid inviability sexual isolation, and hybrid courtship dysfunction. Three regions of the genome were strongly associated with all of these barriers to gene exchange: the left arm of the X-chromosome (XL), the right arm of the X-chromosome (XR), and the center of the second chromosome. The third chromosome contributed to hybrid sterility in one backcross, and the fourth and fifth chromosomes had no detectable association with any of the phenotypes examined. All detectable effects mapped onto the regions that showed inversion differences between these species (XL, XR, center of chromosomes 2 and 3), and the strongest effects were detected in regions that have inversion differences that are fixed within these species (XL and center of chromosome 2).

Our results suggest that much of the genome of D. pseudoobscura and $D$. persimilis may be able to introgress between species via male hybrids (excluding, of course, the completely sterile $F_{1}$ males). Regions that are inverted between these species are the least susceptible to introgression, particularly the XL and second chromosome inversions. However, much of the fourth and fifth chromosomes do not have strong associations with any barriers to gene exchange. Further, the most proximal region of the second chromosome (DPS2003; cytological location in Hamblin and Aquadro 1999) was also not associated with any barrier to gene exchange. Thus, these regions and particularly areas of the fourth and fifth chromosomes should have a high probability of being able to introgress between species when hybridization occurs. This suggestion is consistent with the results of Wang et al. (1997) suggesting that Adh (chromosome 4) has introgressed between these species.

Recombination is effectively inhibited in the areas of the genome that exhibit these inversion differences (Noor and Smith 2000), and single genes with large effects cannot be distinguished from numerous genes with smaller effects. Given that the strongest effects were associated with regions possessing fixed inversion differences, we cannot use standard crosses to further dissect their genetic bases. However, our results suggest that the phenotypes examined may not be both highly polygenic and homogeneously distributed throughout the genome. In particular, some large inverted regions did not possess any detectable effects on several phenotypes studied. If the phenotypes being studied were highly polygenic and distributed homogeneously across the genome, the inverted regions along the XR and third chromosomes should have been associated with at least $11 \%$ and $7 \%$ of the genetic variance, respectively. Given the large sample size used in this study, such differences would have had a high probability of detection. The absence of detected effects associated with such regions suggests either a limited number of genes contribute to these phenotypes or that the genes that contribute to them are clustered in particular genomic regions.

We do not interpret our observation of strong associations between reproductive isolation and the regions inverted between these species to be consistent with chromosomal models of speciation, because only paracentric inversions differentiate these strains. Additionally, $\mathrm{F}_{1}$ and backcross hybrid males possess only one $\mathrm{X}$-chromosome, and thus are not heterozygous for particular chromosomal arrangements. Finally, previous work has identified particular regions within some of these inversion differences that contribute to hybrid sterility, suggesting that the inversions themselves are not responsible (Wu and Beckenbach 1983). 
It may be that regions of the genome close to the large effects we detected in the center of the second chromosome may still be able to introgress between these species. Concurrent work in another laboratory is now identifying patterns of introgression at these same genomic regions, and our combined projects will determine how much linkage to barriers to gene exchange is necessary to prevent gene exchange between $D$. pseudoobscura and D. persimilis. We discuss our current findings regarding the genetic basis of each of the four barriers to gene exchange in turn.

\section{Hybrid Courtship Dysfunction}

We mapped the genetic basis of the hybrid male failure to court (courtship dysfunction) to regions of the $\mathrm{X}$ - and second chromosomes. The left arm of the D. persimilis X-chromosome (XL) had the strongest effect on this phenotype, reducing hybrid male courtship propensity from $85 \%$ to $42 \%$ in BCps males. Our results suggest that genes on the XL interact with loci in or near the second chromosome inversion to produce this failure to court, although other loci may also be involved.

Hybrid behavioral dysfunctions are known in other species (e.g., Buckley 1969; Pashley and Martin 1987; Yoon 1991; Davies et al. 1997), but to our knowledge, this is the first study to identify the nature of the genetic interaction underlying it. In these cases, too, it appears to be restricted to the heterogametic sex, conforming to Haldane's rule (Haldane 1922). Like hybrid male sterility (e.g., Orr 1987; Johnson et al. 1992, 1993), the hybrid male courtship dysfunction in these species is caused at least in part by interactions between the X-chromosome and autosomes. Our observation that its genetic basis resembles that of hybrid sterility justifies claims that it is a behavioral manifestation of Haldane's rule.

We were initially unable to detect autosomal effects on hybrid courtship dysfunction in BCper males, but limiting our dataset to only those males bearing both $D$. persimilis $\mathrm{X}$ chromosomes reduced the background variation such that autosomal effects could be identified. This procedure enhances the probability of detecting QTLs in backcross hybrid males when studying phenotypes caused by epistatic interactions between X-and autosomal loci. Such epistatic interactions appear to be major contributors to barriers to gene exchange, emphasizing the usefulness of this protocol.

\section{Hybrid Sterility}

We found that both arms of the X-chromosome contribute to hybrid male sterility, and interactions with autosomal factors are evident. In addition, we identified a very strong effect of the second chromosome inverted region contributing to the autosomal component of hybrid male sterility. The reduction in BCps male fertility associated with bearing one copy of the D. persimilis allele at DPS2002 was striking (see Table 4). In contrast, loci on the third chromosome, which are also linked to a large inverted region, had a much smaller effect even though the third chromosome inversion (ST/AR) is somewhat larger than the second chromosome inversion (Tan 1935; Dobzhansky and Epling 1944). This contrast suggests either the presence of one or more genes with major effect or a disproportionate number of genes with small ef- fects within the second chromosome inverted region contributing to hybrid male sterility.

Interestingly, we could find no other studies that have documented a specific interaction between an X-chromosomal region and one or more specific autosomal regions causing hybrid sterility, even though such interactions are often assumed to be a major cause of Haldane's rule (for review, see Johnson 2000). Whole chromosome analyses by Dobzhansky (1974) also implicated X-autosome interactions in hybrid sterility among subspecies of $D$. pseudoobscura. Other types of interactions are also sometimes implicated as causes of hybrid sterility (Johnson 2000). Orr (1987) identified a putative $\mathrm{X}-\mathrm{Y}$ incompatibility contributing to sterility in male hybrids of $D$. pseudoobscura and $D$. persimilis, although he noted that " "autosomal genotype is not entirely irrelevant to fertility.", Here, we have identified additional incompatible interactions contributing to hybrid sterility in this species pair.

\section{Hybrid Inviability}

We found alleles at three loci that appeared at frequencies deviating from expectation in adult backcross hybrid males. We inferred that such distortions would result from hybrid inviability rather than meiotic drive because inviability has been previously documented in these species (Weisbrot 1963). However, the distorted segregation switched directions between the two backcrosses. For example, the $D$. persimilis allele at DPSX004 was significantly more prevalent than expected among BCper males, but significantly less prevalent than expected among BCps males. This switch strongly suggests that the distorted frequencies could not have resulted from meiotic drive because the hybrid female genotype that produced the two backcrosses was identical. We conclude that the observed adult allele frequency deviations result from hybrid inviability among backcross hybrid males.

Similar to results from studies of other taxa (Orr et al. 1997; Presgraves and Orr 1998; Orr 1999) and similar to our results on hybrid sterility, we detected an association between $\mathrm{X}$-chromosomal and autosomal loci that contribute to hybrid inviability. In BCper males, hybrid inviability was associated with an interaction between a homozygous $D$. persimilis DPS2002 genotype and a hemizygous D. pseudoobscura Xchromosome. Unlike other well-studied cases of hybrid inviability (e.g., Orr 1993; Coyne et al. 1998), the inviability we observe in this hybridization only appears in the backcross because it involves a homozygous autosomal segment from D. persimilis, whereas $\mathrm{F}_{1}$ hybrids are heterozygous for their autosomes. Deleterious interactions involving homozygous autosomal segments of the genome in hybrids appear to be more common than deleterious interactions involving heterozygous autosomal segments (e.g., Breeuwer and Werren 1995; True et al. 1996) and may generally explain the phenomenon of hybrid breakdown in generations succeeding the $\mathrm{F}_{1}$ generation.

\section{Sexual Isolation}

We found that both $\mathrm{X}$-chromosomal and autosomal loci contribute to hybrid male sexual isolation from both $D$. pseudoobscura and D. persimilis females. With regard to D. pseu- 
doobscura, the XL-chromosome arm contributed greatly to mating success and the XR-chromosome contributed a somewhat lesser, but still significant, effect. The second chromosome inverted region was also moderately associated with mating success to D. pseudoobscura females. With regard to D. persimilis, the XL and second arms contributed significant effects. Thus, the same general genomic regions appear to contribute to both premating and postmating isolation in these species, although it is unlikely that the same genes cause these effects.

In D. pseudoobscura and D. persimilis, sexual isolation results entirely from female species discrimination, so our study has addressed the genetic basis of the preferred male character (Merrell 1954; Noor 1996). A concurrent study has suggested that this preferred male character is their courtship song (Williams et al. 2001), and cuticular hydrocarbon differences do not appear to contribute to the observed sexual isolation (Noor and Coyne 1996). Our results concur with the genetics of courtship song differences in that numerous genes spread across the genome do not cause sexual isolation between these species.

\section{ACKNOWLEDGMENTS}

We thank B. Blanchard, A. Blouin, D. Burkett, A. Hartzog, K. Nettles, K. Rhodes, B. Rogge, T. Rook, N. Tuminello, and M. Williams for technical support. J. Coyne, J. Hey, J. C. Larkin, T. Mackay, and two anonymous reviewers also provided constructive comments on the manuscript and execution of the study. This research was supported by National Institutes of Health (NIH) grant GM58060 subcontracted through J. Hey at Rutgers University to MAFN. YA was supported by a Howard Hughes Medical Institute undergraduate summer research fellowship.

\section{Literature Cited}

Basten, C. J., B. S. Weir, and Z.-B. Zeng. 1999. QTL Cartographer. Vers. 1.14. Department of Statistics, North Carolina State Univ., Raleigh, NC.

Breeuwer, J. A. J., and J. H. Werren. 1995. Hybrid breakdown between two haplodiploid species: the role of nuclear genes and cytoplasmic genes. Evolution 49:705-717.

Buckley, P. A. 1969. Disruption of species-typical behavior patterns in $\mathrm{F}_{1}$ hybrid Agapornis parrots. Zeit. Tierpsychologie 26: 737-747.

Butlin, R. 1998. What do hybrid zones in general, and the Chorthippus parallelus zone in particular, tell us about speciation? Pp. 367-389 in D. J. Howard and S. H. Berlocher, eds. Endless forms: species and speciation. Oxford Univ. Press, New York.

Charlesworth, B., J. A. Coyne, and N. H. Barton. 1987. The relative rates of evolution of sex chromosomes and autosomes. Am. Nat. 130:113-146.

Coyne, J. A. 1984. Genetic basis of male sterility in hybrids between two closely related species of Drosophila. Proc. Natl. Acad. Sci., USA 81:4444-4447.

_ _ 1989. Genetics of sexual isolation between two sibling species, Drosophila simulans and Drosophila mauritiana. Proc. Natl. Acad. Sci. USA 86:5464-5468.

Coyne, J. A., and H. A. Orr. 1989. Two rules of speciation. Pp. 180-207 in D. Otte and J. A. Endler, eds. Speciation and its consequences. Sinauer Associates, Sunderland, MA.

- 1998. The evolutionary genetics of speciation. Phil. Trans. R. Soc. Lond. B 353:287-305.

Coyne, J. A., S. Simeonidis, and P. Rooney. 1998. Relative paucity of genes causing inviability in hybrids between Drosophila melanogaster and D. simulans. Genetics 150:1091-1103.

Darvasi, A., and M. Soller. 1994. Optimum spacing of genetic markers for determining linkage between marker loci and quantitative trait loci. Theor. Appl. Genet. 89:351-357.

Davies, N., A. Aiello, J. Mallet, A. Pomiankowski, and R. E. Silberglied. 1997. Speciation in two neotropical butterflies: extending Haldane's rule. Proc. R. Soc. Lond. B 264:845-851.

Dobzhansky, T. 1936. Studies of hybrid sterility. II. Localization of sterility factors in Drosophila pseudoobscura hybrids. Genetics 21:113-135.

. 1973. Is there gene exchange between Drosophila pseudoobscura and Drosophila persimilis in their natural habitats? Am. Nat. 107:312-314.

- 1974. Genetic analysis of hybrid sterility within the species Drosophila pseudoobscura. Hereditas 77:81-88.

Dobzhansky, T., and C. Epling. 1944. Contributions to the genetics, taxonomy, and ecology of Drosophila pseudoobscura and its relatives. Carnegie Institute of Washington, Washington, D.C.

Dobzhansky, T., and A. H. Sturtevant. 1938. Inversions in the chromosomes of Drosophila pseudoobscura. Genetics 23:28-64.

Doerge, R. W., and G. A. Churchill. 1996. Permutation tests for multiple loci affecting a quantitative character. Genetics 142: 285-294.

Gloor, G. B., and W. R. Engels. 1992. Single-fly DNA preps for PCR. Drosoph. Inf. Serv. 71:148-149.

Gurganus, M. C., S. V. Nuzhdin, J. W. Leips, and T. F. C. Mackay. 1999. High-resolution mapping of quantitative trait loci for sternopleural bristle number in Drosophila melanogaster. Genetics 152:1585-1604.

Haldane, J. B. S. 1922. Sex ratio and unisexual sterility in hybrid animals. J. Genet. 12:101-109.

Hamblin, M. T., and C. F. Aquadro. 1999. DNA sequence variation and the recombinational landscape in Drosophila pseudoobscura: a study of the second chromosome. Genetics 153:859-869.

Hollocher, H. 1998. Reproductive isolation in Drosophila: how close are we to untangling the genetics of speciation? Curr. Op. Gen. Dev. 8:709-714.

Hollocher, H., and C.-I. Wu. 1996. The genetics of reproductive isolation in the Drosophila simulans clade: $X$ vs. autosomal effects and male vs. female effects. Genetics 143:1243-1255.

Jiang, C.-X., P. W. Chee, X. Draye, P. L. Morrell, C. W. Smith, and A. H. Paterson. 2000. Multilocus interactions restrict gene introgression in interspecific populations of polyploid Gossypium (Cotton). Evolution 54:798-814.

Johnson, N. A. 2000. Gene interactions and the origin of species. Pp. 197-212 in J. B. Wolf, E. D. Brodie, and M. Wade, eds. Epistasis and the evolutionary process. Oxford Univ. Press, New York.

Johnson, N. A., D. E. Perez, E. L. Cabot, H. Hollocher, and C.-I. Wu. 1992. A test of reciprocal X-Y interactions as a cause of hybrid sterility in Drosophila. Nature 358:751-753.

Johnson, N. A., H. Hollocher, E. Noonburg, and C.-I. Wu. 1993. The effects of interspecific Y chromosome replacements on hybrid sterility within the Drosophila simulans clade. Genetics 135: 443-453.

Kulathinal, R. J., and R. S. Singh. 2000. A biogeographic approach for testing the role of reinforcement: the case of Drosophila pseudoobscura and D. persimilis. Evolution 54:210-217.

Maside, X. R., and H. F. Naveira. 1996. A polygenic basis of hybrid sterility may give rise to spurious localizations of major sterility factors. Heredity 77:488-492.

Maside, X. R., J. P. Barral, and H. F. Naveira. 1998. Hidden effects of $\mathrm{X}$ chromosome introgressions on spermatogenesis in Drosophila simulans $\times D$. mauritiana hybrids unveiled by interactions among minor genetic factors. Genetics 150:745-754.

Merrell, D. J. 1954. Sexual isolation between Drosophila persimilis and Drosophila pseudoobscura. Am. Nat. 88:93-99.

Noor, M. A. F. 1995. Speciation driven by natural selection in Drosophila. Nature 375:674-675.

_ 1996. Absence of species discrimination in Drosophila pseudoobscura and D. persimilis males. Anim. Behav. 52: 1205-1210. 
1997a. Environmental effects on male courtship intensity in Drosophila pseudoobscura (Diptera: Drosophilidae). J. Insect Behav. 10:305-312.

- $1997 \mathrm{~b}$. Genetics of sexual isolation and courtship dysfunction in male hybrids of Drosophila pseudoobscura and D. persimilis. Evolution 51:809-815.

Noor, M. A. F., and J. A. Coyne. 1996. Genetics of a difference in cuticular hydrocarbons between Drosophila pseudoobscura and D. persimilis. Genet. Res. 68:117-123.

Noor, M. A. F., and K. R. Smith. 2000. Recombination, statistical power, and genetic studies of sexual isolation in Drosophila. J. Hered. 91:99-103.

Noor, M. A. F., N. A. Johnson, and J. Hey. 2000a. Gene flow between Drosophila pseudoobscura and D. persimilis. Evolution 54:2174-2175.

Noor, M. A. F., M. D. Schug, and C. F. Aquadro. 2000b. Microsatellite variation in populations of Drosophila pseudoobscura and Drosophila persimilis. Genet. Res., Camb. 75:25-35.

Orr, H. A. 1987. Genetics of male and female sterility in hybrids of Drosophila pseudoobscura and D. persimilis. Genetics 116: $555-563$

- 1989. Localization of genes causing postzygotic isolation in two hybridizations involving Drosophila pseudoobscura. $\mathrm{He}-$ redity 63:231-237.

. 1993. Haldane's rule has multiple genetic causes. Nature 361:532-533.

. 1999. Does hybrid lethality depend on sex or genotype? Genetics 152:1767-1769.

Orr, H. A., L. D. Madden, J. A. Coyne, R. Goodwin, and R. S. Hawley. 1997. The developmental genetics of hybrid inviability: a mitotic defect in Drosophila hybrids. Genetics 145:1031-1040.

Palumbi, S. R. 1996. Nucleic acids II: the polymerase chain reaction. Pp. 205-247 in D. M. Hillis, C. Moritz, and B. K. Mable, eds. Molecular systematics. Sinauer Associates, Sunderland, MA.

Pashley, D. P., and J. A. Martin. 1987. Reproductive incompatibility between host strains of the fall armyworm (Lepidoptera: Noctuidae). Ann. Ent. Soc. Am. 80:731-733.

Powell, J. R. 1983. Interspecific cytoplasmic gene flow in the absence of nuclear gene flow: evidence from Drosophila. Proc. Natl. Acad. Sci. USA 80:492-495.

_ - 1991. Monophyly/ paraphyly/ polyphyly and gene/ species trees: an example from Drosophila. Mol. Biol. Evol. 8:892-896.
Presgraves, D. C., and H. A. Orr. 1998. Haldane's rule in taxa lacking a hemizygous X. Science 282:952-954.

Rice, W. R. 1989. Analyzing tables of statistical tests. Evolution 43:223-225.

Rieseberg, L. H., J. Whitton, and K. Gardner. 1999. Hybrid zones and the genetic architecture of a barrier to gene flow between two sunflower species. Genetics 152:713-727.

Ritchie, M. G., and S. D. F. Phillips. 1998. The genetics of sexual isolation. Pp. 291-308 in D. J. Howard and S. H. Berlocher, eds. Endless forms: species and speciation. Oxford Univ. Press, New York.

Sturtevant, A. H., and T. Dobzhansky. 1936. Geographical distribution and cytology of "Sex Ratio" in Drosophila pseudoobscura and related species. Genetics 21:473-490.

Tan, C. C. 1935. Salivary gland chromosomes in the two races of Drosophila pseudoobscura. Genetics 20:392-402.

. 1946. Genetics of sexual isolation between Drosophila pseudoobscura and Drosophila persimilis. Genetics 31:558-573.

Ting, C.-T., S.-C. Tsaur, M.-L. Wu, and C.-I. Wu. 1998. A rapidly evolving homeobox at the site of a hybrid sterility gene. Science 282:1501-1504

True, J. R., B. S. Weir, and C. C. Laurie. 1996. A genome-wide survey of hybrid incompatibility factors by the introgression of marked segments of Drosophila mauritiana chromosomes into Drosophila simulans. Genetics 142:819-837.

Wang, R. L., J. Wakeley, and J. Hey. 1997. Gene flow and natural selection in the origin of Drosophila pseudoobscura and close relatives. Genetics 147:1091-1106.

Weisbrot, D. R. 1963. Studies on differences in the genetic architecture of related species of Drosophila. Genetics 48:1121-1139.

Williams, M. A., A. G. Blouin, and M. A. F. Noor. 2001. Courtship songs of Drosophila pseudoobscura and D. persimilis. II. Genetics of species differences. Heredity 86: In press.

Wu, C.-I., and A. T. Beckenbach. 1983. Evidence for extensive genetic differentiation between the sex-ratio and the standard arrangement of Drosophila pseudoobscura and D. persimilis and identification of hybrid sterility factors. Genetics 105:71-86.

Wu, C.-I., and M. F. Palopoli. 1994. Genetics of postmating reproductive isolation in animals. Annu. Rev. Genet. 27:283-308.

Yoon, C. K. 1991. Molecular and behavioral evolution in the semispecies of Drosophila athabasca. Ph.D. diss., Cornell University, Ithaca, NY.

Corresponding Editor: H. A. Orr 